\title{
Evolution of Magnetic and Mechanical Properties in FINEMET-Type Precursor Glasses during Isothermal and Pulse Heat Treatments
}

\author{
L. HubaČ $\check{c}^{a *}$, L. NovÁK ${ }^{a}$, S. BAlla ${ }^{b}$ AND A. LOVAS ${ }^{b}$ \\ ${ }^{a}$ Department of Physics, Technical University of Košice, Park Komenského 2, 04200 Košice, Slovakia \\ ${ }^{b}$ Department of Automobiles and Vehicle Manufacturing, Budapest University of Technology and Economics, \\ Stoczek u.2., 1111 Budapest, Hungary
}

The effects on the evolution of coercivity $\left(H_{c}\right)$ and brittleness in FINEMET-type precursor glasses during traditional (isothermal) and pulse heat treatments are compared. The time and temperature dependences of these quantities are monitored during the series of isothermal and pulse heat treatments. The activation energy for $H_{c}$ relaxation is lower than that for the brittleness evolution, however, dramatic changes in the values of these quantities can be detected above $300^{\circ} \mathrm{C}$. Both quantities change mainly with temperature, they are less timedependent.

DOI: 10.12693/APhysPolA.131.672

PACS/topics: $75.50 . \mathrm{Kj}, 75.30 . \mathrm{Gw}, 75.70 .-\mathrm{i}$

\section{Introduction}

The significance of FINEMET-type nanocrystalline alloys is based on their outstanding permeability, coupled with satisfactory saturation magnetization. Their properties are especially promising in high frequency applications $[1,2]$. Additional advantage of these alloys is a wide range of possibilities for tailoring the shape of magnetization curve enabling also extended applications. Besides traditional nanocrystallization heat treatments, field and pulse as well as stress-annealings have also been proposed recently for tailoring their magnetization processes $[3,4]$. During traditional, isothermal annealings (leading to amorphous-nanocrystalline transformation) the deterioration of mechanical properties of glassy ribbons are often observed, which can prevent further magnetic applications. In the present paper the evolution of magnetic softening ( $H_{c}$ decrease) and the development of mechanical embrittlement, after isothermal and pulse heat treatments are reported. The process of coercive field decrease as well as the evolution of brittleness are monitored.

\section{Experimental}

The measurements were performed on FINEMET-type $\left(\mathrm{Fe}_{73.5} \mathrm{Cu}_{1} \mathrm{Nb}_{3} \mathrm{Si}_{13.5} \mathrm{~B}_{9}\right)$ glassy samples (nanocrystalline precursor alloys) in computer-controlled magnetometer. Isothermal and pulse heat treatments resulted in either structural relaxation, or crystallization. Complete thermomagnetic curves were measured on the samples after

\footnotetext{
*corresponding author; e-mail: lukas.hubac@tuke.sk
}

heat treatments and the curves obtained from the different series of pulse heat treatments were compared. The experimental setup for pulse heating is described in [5]. This configuration allows pulse heating as well as measuring the magnetic polarization of the sample in the process of pulse heating.

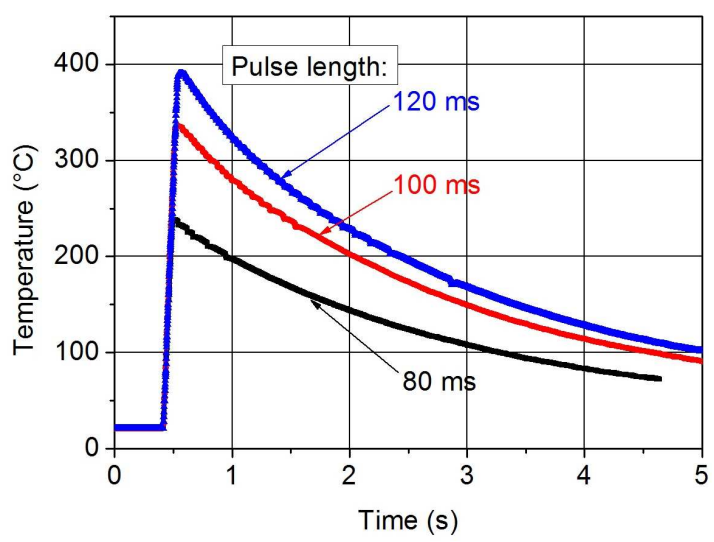

Fig. 1. The temperature versus time dependence for pulses of different length, measured on FINEMET sample.

The peak temperatures, measured on FINEMET sample by applying pulses of different length are plotted in Fig. 1. One can see that pulses provide high temperature over a very short time interval (typically $1 \mathrm{~s}$ ) over the peak. The maximum peak temperature depends mainly on the pulse length. Temperature calibration was performed by measuring the thermomagnetic curve on the same samples. The Curie temperature which is known for this alloy was determined using a vibrating sample magnetometer. The isothermal heat treatments were performed in resistance furnace under protective atmosphere. Typical duration of heat treatment was $1 \mathrm{~h}$. 


\section{Results and discussion}

\subsection{The evolution of $H_{c}$ during pulse heat treatments}

It is well known that coercivity of glassy soft magnetic ribbons decreases when the samples are subject to heat treatments. This phenomenon is known as stress (structural) relaxation. Coercivity in the as-quenched state originates from several factors like intrinsic fluctuations of local anisotropies, clusters of chemical shortrange ordered regions, surface irregularities (surface and volume pinning of domain walls). All kinds of irregularities may act as pinning centres for the domain wall displacements [6]. The impact of these factors is modified by heat treatments: the change of surface irregularities (roughness, segregated oxide inclusions) are not expected to change during the stress relieve. In contrast, the short range order-scale clusters, together with the possible bond-reorientation between the neighbouring metalmetalloid atoms may occur, without formation of traces of any crystalline nuclei. First the $H_{c}$ evolution in the studied samples during pulse and isothermal heat treaments is compared.

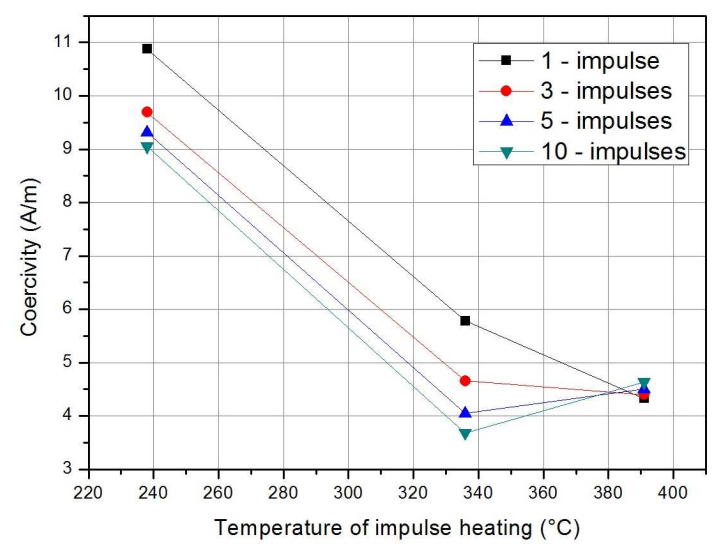

Fig. 2. Change of the coercivity $H_{c}$ versus the peak temperature of pulses for various pulse numbers.

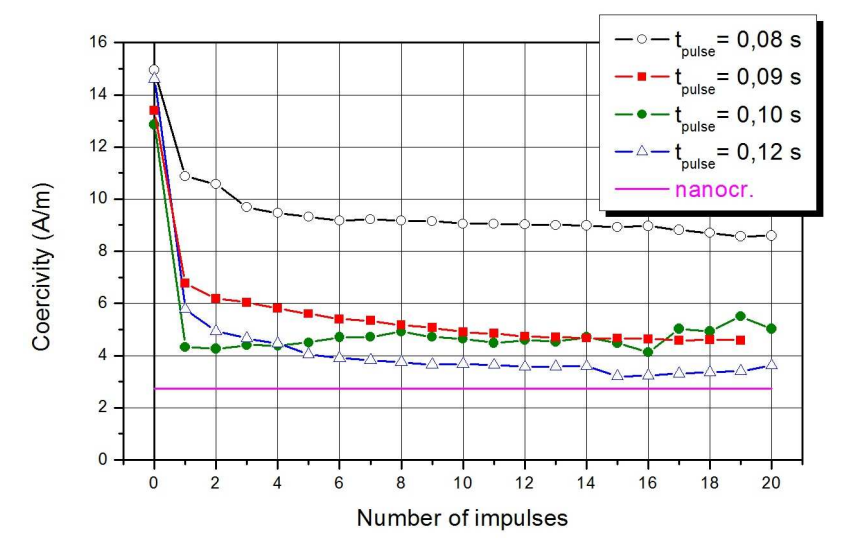

Fig. 3. Coercivity $H_{c}$ versus the number of pulses with different pulse length.

In Fig. 2 the trend of $H_{c}$ change is illustrated for different series of pulses, versus the peak temperature (deter- mined on the basis of Fig. 1). The increased number of heat pulses results in only slight drop of $H_{c}$ below $300^{\circ} \mathrm{C}$ (compare the points referred to $1,3,5$, and 10 pulses at $\left.T=240^{\circ} \mathrm{C}\right)$. Therefore, the $H_{c}$ increase versus the peak number exhibits saturation at low temperature. On the other hand, the $H_{c}$ values are within the interval of experimental error, when the peak temperature approaches $400^{\circ} \mathrm{C}$. It means that characteristic activation energy is required to attain a fully "relaxed state" and this state can be slowly obtained by the series of "low temperature" pulses.

This trend is also supported by results in Fig. 3 in which the $H_{c}$ is plotted versus the series of very short pulses $(0.06,0.07$, and $0.08 \mathrm{~s})$. In this experiment $240^{\circ} \mathrm{C}$ was achieved using $0.08 \mathrm{~s}$ pulse length. The abrupt decrease of $H_{c}$ due to a single thermal shock is surprising. This value approaches that one obtained during $1 \mathrm{~h}$ isothermal heat treatment (see also Fig. 3). From this figure it is also clear that even the lowest pulse length applied can induce detectable $H_{c}$ relaxation, however, this $H_{c}$ change exhibits stepwise character, showing the limit of $H_{c}$ relaxation at this temperature. Similar trends were observed by measuring $K_{i}$ after the outlined pulse heat treatments.

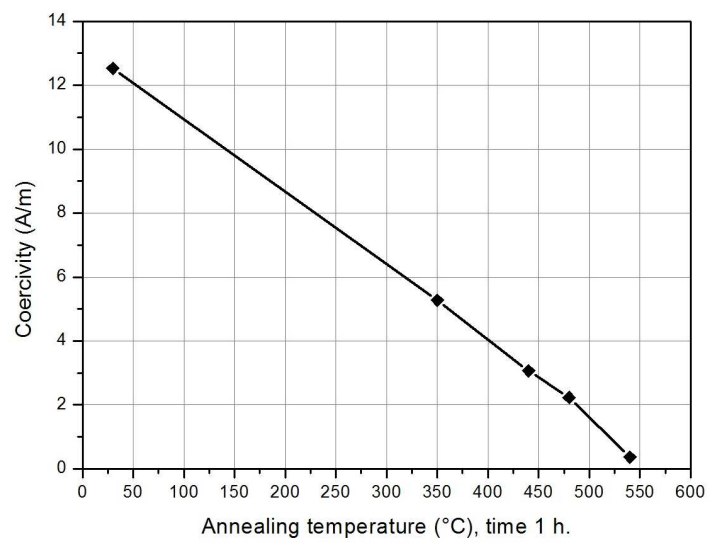

Fig. 4. Dependence of $H_{c}$ on the temperature of isothermal annealings in FINEMET-type precursor.

\subsection{Isothermal heat treatments}

Similar trend in $H_{c}$ decrease is found with increasing temperature of annealing $T$ (Fig. 4). Comparing the $H_{c}(T)$ trends, illustrated in Fig. 3 and 4, comparing the pulse and isothermal heat treatments, one can conclude that more than $50 \%$ of $H_{c}$ decrease arises from structural relaxation of glassy state and the rest is due to the development of nanocrystalline grain system, i.e. due to the anisotropy averaging within the distance of domain wall thickness [7].

\subsection{The evolution of brittleness during pulse annealings}

Typical results on the evolution of brittleness $\varepsilon_{f}$ (defined in [8]) are shown in Fig. 5. The $\varepsilon_{f}$ values are plotted versus the number of pulses with increasing 
length. It is remarkable that when applying $0.08 \mathrm{~s}$ pulse length $\left(240^{\circ} \mathrm{C}\right.$ peak temperature $)$ the flexibility of the as-quenched precursor is unchanged, even over 20 pulses. In contrast, the $H_{c}$ decreases more than $70 \%$ already due to the first few pulses, showing the difference in the activation energy and the mechanism of processes involved. Another important feature of pulse annealings is that brittleness is developed very rapidly exceeding a critical pulse length (critical peak temperature), as it is illustrated in Fig. 5.

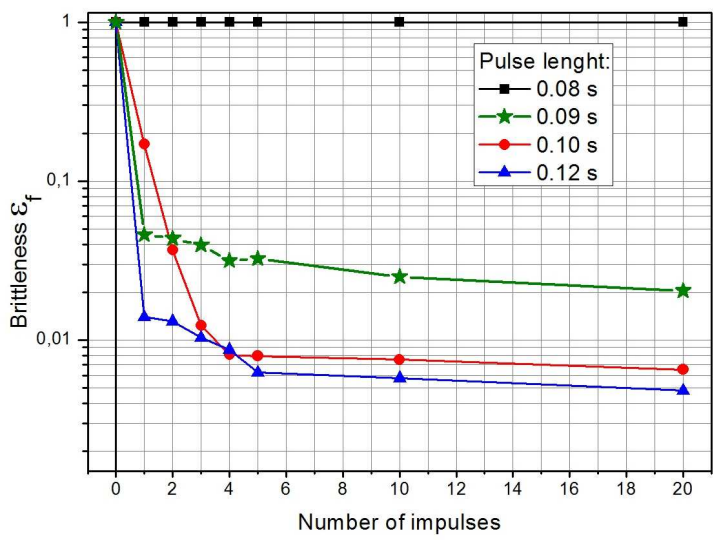

Fig. 5. The change in brittleness (defined in [8]) versus the number of pulses with increasing length.

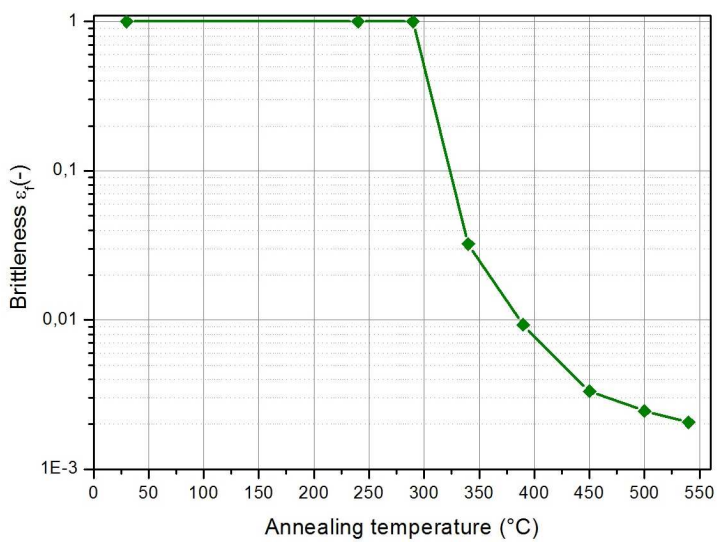

Fig. 6. Brittleness of FINEMET ribbons after isothermal heat treatments at various temperatures.

\subsection{Comparison of brittleness of samples after pulse and isothermal heat treatments}

Comparing the flexible-brittle transitions in samples after pulse and isothermal heat treatments (compare Figs. 5 and 6), one can notice that temperature limit between the two region is nearly the same for both type of heat treatments - approximately $300^{\circ} \mathrm{C}$. Below this temperature the plastic behaviour is nearly the same. Nevertheless, the degree of brittleness is much higher in samples subject to isothermal annealings. It means that sensitivity to bending (shear strength) is better for samples after pulse heat treatments.

\section{Conclusions}

1. The evolutions of coercivity and brittleness in FINEMET-type glassy samples are compared during pulse and traditional isotermal heat treatments over the period of structural relaxation and nanograin formation.

2. The coercivity decrease represents the degree of relaxation, depending mainly on heat treatment temperature (i.e., peak temperature in the case of pulse annealings) while the duration of isothermal heat treatment is of secondary importance.

3. In the case of pulse heat treatments the role of peak (pulse peak) has a great influence, while the pulse number is of secondary importance for achieved degree of relaxation.

4. The activation energy of $H_{c}$ relaxation is lower than that for the brittleness evolution.

5. Dramatic decrease in $H_{c}$ and flexibility breakdown are observed above $300{ }^{\circ} \mathrm{C}$ in both type of heat treatments, however, the degree of brittleness after isothermal annealings is three times higher.

\section{Acknowledgments}

This work was supported by the project VEGA $1 / 0413 / 15$ of the Scientific Grant Agency of the Ministry for Education of the Slovak Republic and also by the project "Centre of Excellence for Integrated Research \& Exploitation of Advanced Materials and Technologies in Automotive Electronics" ITMS 26220120055 .

\section{References}

[1] M. Makino, A. Inoue, T. Matsumoto, Mater. Trans. JIM 36, 924 (1995).

[2] G. Herzer, in: Handbook of Magnetic Materials, Vol. 10, Ed. K.H.J. Buschow, Elsevier Sci., Amsterdam 1997, Ch. 3, p. 415.

[3] A. Lovas, L. Hubač, L. Novák, Acta Phys. Pol. A 126, 100 (2014).

[4] Y. Iwami, Y. Okazaki, T. Shimizu, T. Hirakawa, A. Saito, J. Magn. Magn. Mater. 215-216, 443 (2000).

[5] J. Kováč, L. Novák, L. Hubač, J. Electr. Eng. 66, $142(2015)$.

[6] F.E. Luborsky, in: Amorphous Metallic Alloys, Ed. F.E. Luborsky, Monographs in Materials, Butterworths, London 1984, p. 360.

[7] G. Herzer, L.K. Varga, J. Magn. Magn. Mater. 215-216, 506 (2000).

[8] R. Gerling, R. Wagner, J. Nucl. Mater. 107, 311 (1982). 